\title{
Status of the ESS neutrino Super Beam
}

\author{
Marcos Dracos ${ }^{* \dagger}$ \\ IPHC, Université de Strasbourg, CNRS/IN2P3 \\ F-67037 Strasbourg, France \\ E-mail: marcos.dracosein2p3.fr
}

\begin{abstract}
After measuring in 2012 a relatively large value of the neutrino mixing angle $\theta_{13}$, the door is now open to observe for the first time a possible $\mathrm{CP}$ violation in the leptonic sector. The measured value of $\theta_{13}$ also privileges the 2 nd oscillation maximum for the discovery of $\mathrm{CP}$ violation instead of the usually used 1st oscillation maximum. The sensitivity at this 2 nd oscillation maximum is about three times higher than for the 1st oscillation maximum inducing a lower influence of systematic errors. Going to the 2nd oscillation maximum necessitates a very intense neutrino beam with the appropriate energy. The world's most intense pulsed spallation neutron source, the European Spallation Source, will have a proton linac with $5 \mathrm{MW}$ power and $2 \mathrm{GeV}$ energy. This linac, under construction, has the potential to become the proton driver of the world's most intense neutrino beam with very high potential to discover a neutrino $\mathrm{CP}$ violation. The physics performance of that neutrino Super Beam in conjunction with a megaton underground Water Cherenkov neutrino detector installed at a distance of about $500 \mathrm{~km}$ from ESS has been evaluated. In addition, the choice of such detector will extent the physics program to proton-decay, atmospheric neutrinos and astrophysics searches. The ESS proton linac upgrades, the accumulator ring needed for proton pulse compression, the target station optimization and the physics potential are described. In addition to neutrinos, this facility will also produce at the same time a copious number of muons which could be used by a low energy nuSTORM facility, a future Neutrino Factory or a muon collider. The ESS neutron facility will be fully ready by 2023 at which moment the upgrades for the neutrino facility could start.

This project is now supported by the COST Action CA15139 "Combining forces for a novel European facility for neutrino-antineutrino symmetry-violation discovery" (EuroNuNet).
\end{abstract}

The 19th International Workshop on Neutrinos from Accelerators-NUFACT2017

25-30 September, 2017

Uppsala University, Uppsala, Sweden

\footnotetext{
*Speaker.

${ }^{\dagger}$ On behalf of the ESS $v$ SB/EuroNuNet project.
} 


\section{Introduction}

In neutrino oscillations two main parameters remain unmeasured, the neutrino mass hierarchy (which neutrino is the lightest one) and a possible CP violation in the leptonic sector described by the PMNS phase parameter $\delta_{C P}$. Since the measurement in 2012 of the last neutrino oscillation angle $\theta_{13}[1,2,3]$, which has been found to be relatively large, the accessibility to the above parameters became easier than what it was believed previously.

For the mass hierarchy determination, it became possible to use medium baseline reactor neutrino experiments $[4,5,6,7,8]$ or/and ice or sea experiments using atmospheric neutrinos [10, 9]. Experiments are already in preparation going in this direction $[11,12]$ and it is expected that the mass hierarchy issue will be solved before the end of next decade.

For the observation of a possible $\mathrm{CP}$ violation in the leptonic sector very intense neutrino beams are needed. To achieve this, multi-MW power proton beams are necessary accompanied by many sever operation conditions due to the very high power. Due to the relatively large $\theta_{13}$ value, it came out that by operating the neutrino facilities at the second oscillation maximum (instead of the first oscillation maximum), more sensitivity to $\delta_{C P}$ is acquired while being less sensitive to systematic errors [13, 14, 15]. The European Spallation Source [17] (ESS) under construction in Lund, for neutron production, will use a $5 \mathrm{MW}$ proton linac which could also be used to produce a very powerful neutrino beam. This project called ESSvSB [18] (for ESS neutrino Super Beam) is described in this paper.

\section{The European Spallation Source Linac}

The ESS facility under construction in Lund, Sweden, since 2014, will provide to the European community a very powerful neutron facility for many applications. The whole installation with all neutron stations will be fully operational around 2023 . To produce spallation neutrons this facility will use a very powerful proton linac (Fig. 1) with an average beam power of $5 \mathrm{MW}$, a peak power of $125 \mathrm{MW}$ and a duty cycle of only $4 \%$ (pulse duration of $2.86 \mathrm{~ms}$ ).

A way to also produce a very powerful neutrino beam using the same linac is to double the pulse frequency from $14 \mathrm{~Hz}$ to $28 \mathrm{~Hz}$, thus, doubling the average proton power. By alternating the pulses between the two facilities, neutrons and neutrinos could be produced at the same time. In this case, the number of protons on target per year would be for each facility of the order of $2.7 \times 10^{23}$. The proton kinetic energy will be $2.0 \mathrm{GeV}$. It could go up to $3.6 \mathrm{GeV}$ in future upgrades using empty space left in purpose in the linac tunnel.

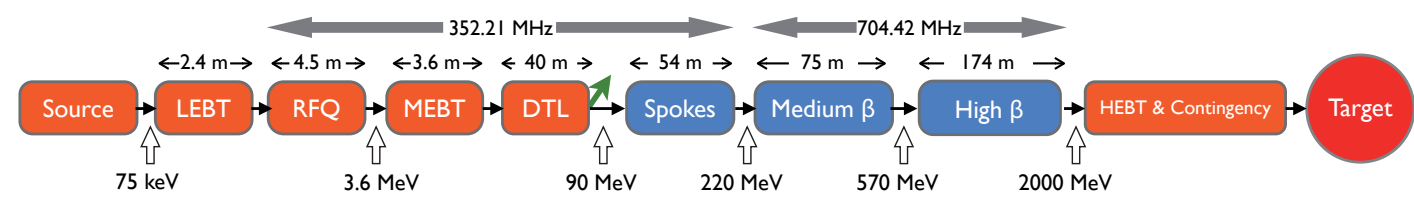

Figure 1: Schematic view of the ESS proton linac.

By sending this proton beam against a target and using a hadron collector as described below the neutrino/antineutrino un-oscillated spectra depicted by Fig. 2 can be obtained at an arbitrary 
distance of $100 \mathrm{~km}$ from the target for 200 operational days (one year). Table 1 presents the number of neutrinos corresponding to Fig. 2. It has to be noted that the total contamination is very weak, especially the one coming from electron neutrinos/antineutrinos which is of the order of $0.5 \%$. These electron neutrinos/antineutrinos which will disturb the physics performance of the project studying the $v_{\mu} \rightarrow v_{e}$ oscillation, could be used to measure the electron neutrino/antineutrino crosssections in an appropriate near detector and thus reduce further the systematic errors of the project.
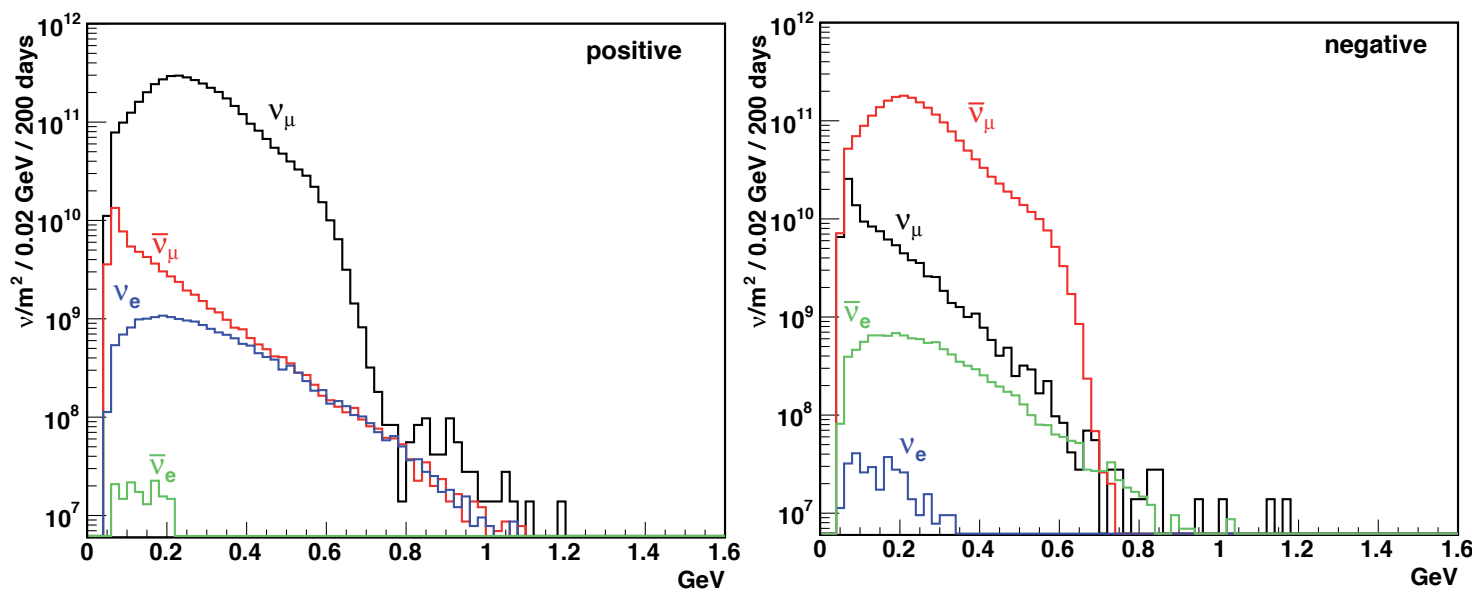

Figure 2: Neutrino energy spectrum for "neutrinos" (left, positive current in the horn) and "antineutrinos" (right, negative current in the horn) at a distance of $100 \mathrm{~km}$ on-axis from the target station, for $2.0 \mathrm{GeV}$ protons.

Table 1: Number of neutrinos per $\mathrm{m}^{2}$ crossing a surface placed on-axis at a distance of $100 \mathrm{~km}$ from the target station during 200 days for $2.0 \mathrm{GeV}$ protons and positive and negative horn current polarities.

\begin{tabular}{|c|c|c|c|c|}
\hline \multirow{2}{*}{} & \multicolumn{2}{|c|}{ positive } & \multicolumn{2}{c|}{ negative } \\
\cline { 2 - 5 } & $N_{v}\left(\times 10^{10}\right) / \mathrm{m}^{2}$ & $\%$ & $N_{v}\left(\times 10^{10}\right) / \mathrm{m}^{2}$ & $\%$ \\
\hline$v_{\mu}$ & 396 & 97.9 & 11 & 1.6 \\
\hline $\bar{v}_{\mu}$ & 6.6 & 1.6 & 206 & 94.5 \\
\hline$v_{e}$ & 1.9 & 0.5 & 0.04 & 0.01 \\
\hline $\bar{v}_{e}$ & 0.02 & 0.005 & 1.1 & 0.5 \\
\hline
\end{tabular}

\section{2nd Oscillation maximum}

To observe a possible CP violation in the leptonic sector the neutrino oscillations $v_{\mu} \rightarrow v_{e}$ and $\bar{v}_{\mu} \rightarrow \bar{v}_{e}$ have to be studied and compared. The oscillation probability has mainly three terms, commonly called "atmospheric", "solar" and "interference":

$$
\begin{array}{rlrl}
P_{\left(v_{\mu} \rightarrow v_{e}\right)\left(\bar{v}_{\mu} \rightarrow \bar{v}_{e}\right)} \approx & \sin ^{2} \theta_{23} \sin ^{2} 2 \theta_{13}\left(\frac{\Delta_{13}}{\tilde{B}_{\mp}}\right)^{2} \sin ^{2}\left(\frac{\tilde{B}_{\mp} L}{2}\right) & & \text { atmospheric } \\
& -\cos ^{2} \theta_{23} \sin ^{2} 2 \theta_{12}\left(\frac{\Delta_{12}}{A}\right)^{2} \sin ^{2}\left(\frac{A L}{2}\right) & & \text { solar } \\
& +\tilde{J} \frac{\Delta_{12}}{A} \frac{\Delta_{13}}{\tilde{B}_{\mp}} \sin \left(\frac{A L}{2}\right) \sin \left(\frac{\tilde{B}_{\mp} L}{2}\right) \cos \left( \pm \delta_{C P}-\frac{\Delta_{13} L}{2}\right) & \text { interference }
\end{array}
$$


with $\tilde{J}=\cos \theta_{13} \sin 2 \theta_{12} \sin 2 \theta_{23} \sin 2 \theta_{13}, \Delta_{i j}=\frac{\Delta_{i j}^{2}}{2 E_{v}}, \tilde{B}_{\mp}=\left|A \mp \Delta_{13}\right|$ and $A=\sqrt{2} G_{F} N_{e}$ (with $N_{e}$ the electron density, the parameter taking into account matter effects). The interference term carries the $\mathrm{CP}$ violating parameter $\delta_{C P}$ and must be as high as possible compared to the other two terms to avoid being affected by the associated systematic errors.

Neglecting matter effects, the matter/antimatter asymmetry is defined as:

$$
\mathscr{A}=\frac{P_{v_{\mu} \rightarrow v_{e}}-P_{\bar{v}_{\mu} \rightarrow \bar{v}_{e}}}{P_{v_{\mu} \rightarrow v_{e}}+P_{\bar{v}_{\mu} \rightarrow \bar{v}_{e}}}
$$

For $\theta_{13} \sim 8^{\circ}$, the interference term dominates the two other terms at a position corresponding to the second oscillation maximum instead of the first one [14]. Moreover, at the second oscillation maximum the matter/antimatter asymmetry is of the order of $\mathscr{A}=0.75 \sin \delta_{C P}$ while at the first oscillation maximum this is only $\mathscr{A}=0.3 \sin \delta_{C P}$ [15], procuring significantly more sensitivity to observe a possible $\mathrm{CP}$ violation. The drawback is that, for the same neutrino energy, the project baseline has to be about three times higher than for the first oscillation maximum, significantly decreasing the statistics. For this, a very intense neutrino beam is needed necessitating a multiMW proton beam.

\section{The neutrino facility}

In order to use the ESS proton beam an accumulator ring has to be used to shorten the proton pulses from $2.86 \mathrm{~ms}$ to few $\mu \mathrm{s}$. This requirement comes from the fact that the hadron collector (magnetic horn), used to focus hadrons before decaying to neutrinos, cannot afford very long current pulses $(\sim 350 \mathrm{kA})$. Also, the use of an accumulator necessitates to accelerate $\mathrm{H}^{-}$instead of protons because of charge effects, increasing further the cost of the project.

The protons exiting the accumulator are sent on a target followed by a magnetic horn. The hadrons (mainly pions) decay in a tunnel producing neutrinos and muons before hitting a beam dump. The target station including the beam dump could be similar to the one designed by the EUROv Design Study [16]. In this configuration the target station consists of four targets placed inside four horns, hit alternatively by the proton beam in order to mitigate the very high power effect.

Fig. 3 shows a schematic view of the target station. It has to be noted that the decay tunnel length is only $25 \mathrm{~m}$ allowing the possibility to fulfil all volume of the target station with He avoiding the presence of decay tunnel wall after the magnetic horns.

Fig. 4 presents the whole neutron/neutrino facility. A near detector could be placed at about $500 \mathrm{~m}$ after the target station to monitor the neutrino flux and measure neutrino cross-sections relevant to the ESSvSB physics program. A Mton Water Cherenkov detector is proposed to be placed in the north of Lund at about a distance of $500 \mathrm{~km}$ to detect the neutrinos after oscillation.

\section{Physics Performance}

For the evaluation of the physics performance to observe a $\mathrm{CP}$ violation in the leptonic sector it is assumed to have MEMPHYS [19, 20] as far detector placed in the area of Garpenberg active mine at $540 \mathrm{~km}$ from Lund. In this configuration and using the horn shape defined by EUROv 


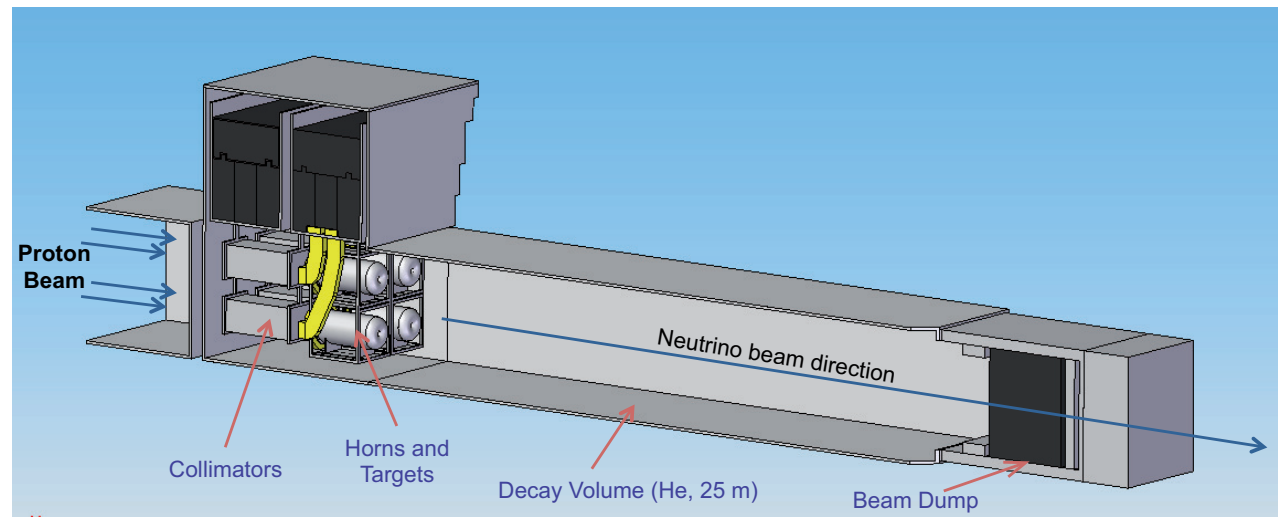

Figure 3: ESS $v$ SB Target Station and decay tunnel.

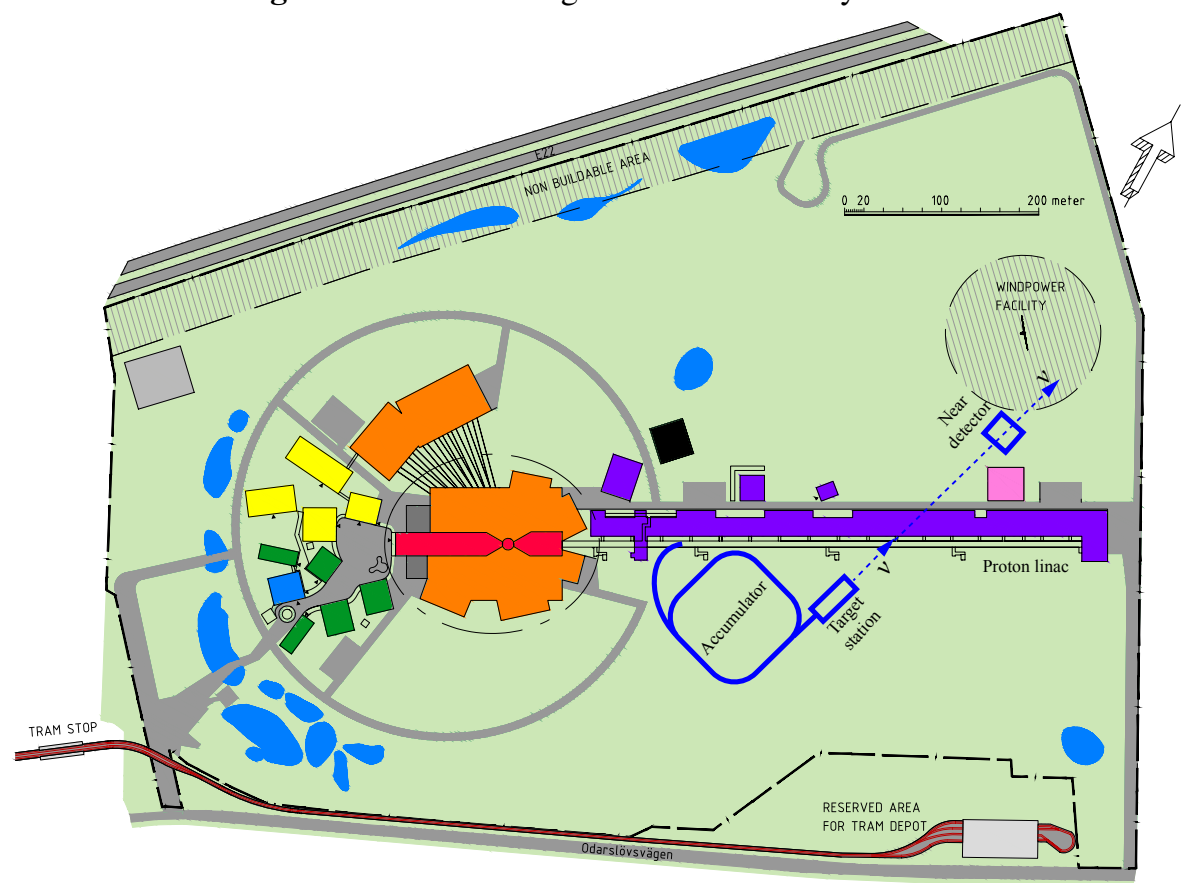

Figure 4: The ESS neutron and neutrino (in blue) facilities.

without further optimisations, the second oscillation is well covered. Fig. 5 presents the $v_{\mu} \rightarrow v_{e}$ oscillation probability as function of the neutrino energy. On this distribution it is superimposed the energy spectrum of electron neutrinos detected by the far detector after oscillation. It is well seen that this facility is almost exclusively operated at the second oscillation maximum.

Fig. 6 [21] presents the significance to discover a $\mathrm{CP}$ violation as a function of the $\delta_{C P}$ parameter. It has to be recalled that a matter/antimatter asymmetry is observed if this parameter is different from zero and $180^{\circ}$. For these calculations it has been assumed a systematic error of $5 \%$ for the signal evaluation and $10 \%$ for the background. The figure presents two possible scenarios of data taking, one corresponding to 2 years of neutrinos and 8 years of anti-neutrinos and the second one for 5 years neutrinos and 5 years anti-neutrinos. The second scenario seems slightly better. Fig. 7 shows the discovery significance as a function of the fraction of the full $\delta_{C P}$ range. For a significance corresponding to $5 \sigma$ more than $60 \%$ of the $\delta_{C P}$ values can be covered. 


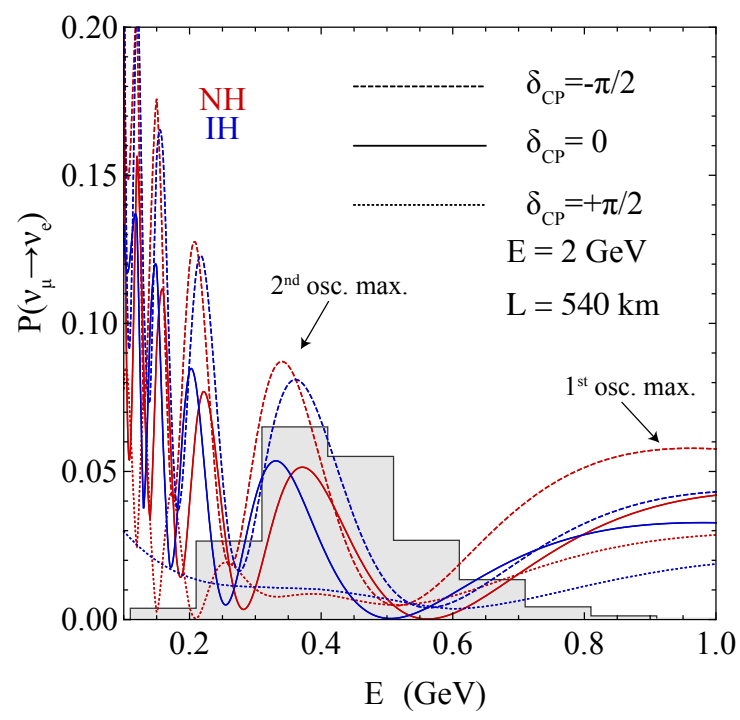

Figure 5: Oscillation probability and electron neutrino energy spectrum (gray area).

The $\mathrm{CP}$ violation discovery performance of this facility is almost not affected by the unknown neutrino mass hierarchy because of the relatively short baseline not inducing significant matter effect. The mass hierarchy could be determined using together the accelerator and atmospheric neutrinos with a significance of more than $5 \sigma$. But, it is believed that this issue will be solved by reactor and atmospheric neutrino experiments before the operation of this facility.

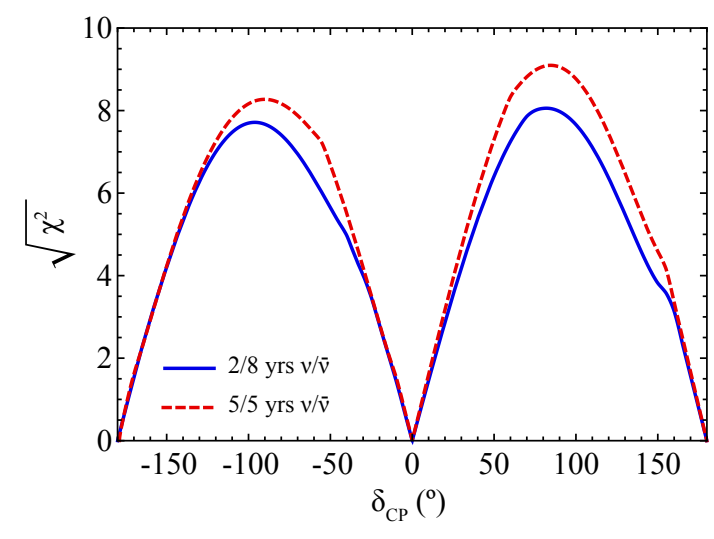

Figure 6: The significance to discover a $\mathrm{CP}$ violation as a function of the $\delta_{C P}$ parameter.

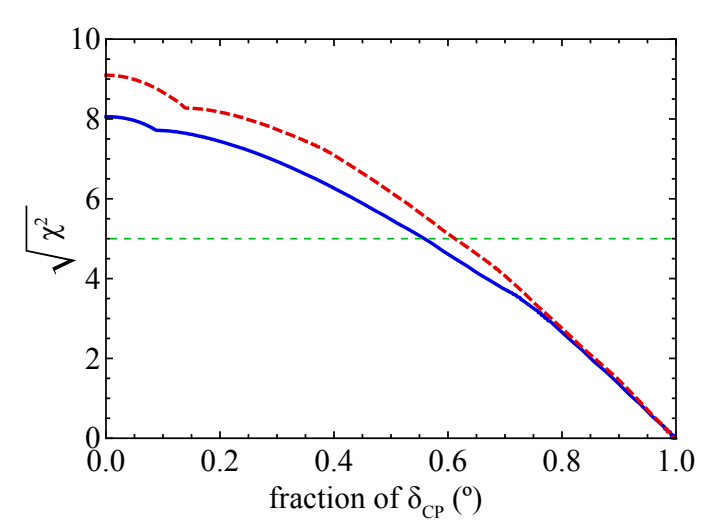

Figure 7: The significance in terms of number of standard deviations with which $\mathrm{CP}$ violation can be discovered as function of the fraction of the full $\delta_{C P}$ range.

\section{Muon potentiality}

Together with the neutrino production the ESS $v$ SB facility would also produce from pion decays a huge number of muons. These muons could be collected at the level of the beam dump using specific devices and used for other applications. The mean momentum of the muons is of 
the order of $0.46 \mathrm{MeV}$. A magnet and a collecting device could be placed at the level of the beam dump to deviate and collect a significant fraction of these muons. Preliminary calculations show that more than $4 \times 10^{20}$ muons per year can be extracted. These muons can be used for a "low" nuSTORM neutrino experiments [22], for a Neutrino Factory and for R\&D for 6D muon cooling and studies for a possible future muon collider.

\section{European Projects}

Since 2016, the COST Action (networking) CA15139/EuroNuNet [23] supports this project and all activities in Europe on CP violation discovery in the neutrino sector. This COST Action which will last up to 2020 gathers up to now 13 European countries. In 2017 a European H2020 INFRADEV application has been accepted for a duration of four years in order to perform a design study of the ESSvSB project and produce a Conceptual Design Report. This project has a cost of 4.7 M€, $3 \mathrm{M} €$ being provided by EU.

\section{Conclusions}

For a relatively high value of the neutrino oscillation angle $\theta_{13}$ as the one measured these last years, the sensitivity to a possible $\mathrm{CP}$ violation in the neutrino sector is significantly higher at the second oscillation maximum than the first one. Also, measurements at the second oscillation maximum are less affected by systematic errors.

In order to operate a neutrino facility with a baseline corresponding to the second oscillation maximum a multi-MW proton driver is needed. The European Spallation Source will have in few years a $5 \mathrm{MW}$ proton linac with a duty cycle of only $4 \%$. By doubling the frequency of the linac another $5 \mathrm{MW}$ proton beam can be obtained for a neutrino facility operated in parallel with the neutron facility.

Using the ESS linac, an adequate target station and a Mton far detector, up to $60 \%$ of $\delta_{C P}$ space can be covered for a CP violation discovery at the $5 \sigma$ level. This is proposed by the ESS $v$ SB project supported by the COST Action CA15139 and the corresponding EU H2020 Design Study.

\section{Acknowledgment}

This project is supported by the COST Action CA15139 "Combining forces for a novel European facility for neutrino-antineutrino symmetry-violation discovery" (EuroNuNet).

\section{References}

[1] Y. Abe et al. [DOUBLE-CHOOZ Collaboration], "Indication for the disappearance of reactor electron antineutrinos in the Double Chooz experiment," Phys. Rev. Lett. 108, 131801 (2012) [arXiv:1112.6353 [hep-ex]].

[2] F. P. An et al. [DAYA-BAY Collaboration], "Observation of electron-antineutrino disappearance at Daya Bay,” Phys. Rev. Lett. 108, 171803 (2012) [arXiv:1203.1669 [hep-ex]].

[3] J. K. Ahn et al. [RENO Collaboration], "Observation of Reactor Electron Antineutrino Disappearance in the RENO Experiment,” Phys. Rev. Lett. 108, 191802 (2012) [arXiv:1204.0626 [hep-ex]]. 
[4] S. T. Petcov and M. Piai, "The LMA MSW solution of the solar neutrino problem, inverted neutrino mass hierarchy and reactor neutrino experiments," Phys. Lett. B 533 (2002) 94 doi:10.1016/S0370-2693(02)01591-5 [hep-ph/0112074].

[5] S. Choubey, S. T. Petcov and M. Piai, "Precision neutrino oscillation physics with an intermediate baseline reactor neutrino experiment," Phys. Rev. D 68 (2003) 113006 doi:10.1103/PhysRevD.68.113006 [hep-ph/0306017].

[6] J. Learned, S. T. Dye, S. Pakvasa and R. C. Svoboda, "Determination of neutrino mass hierarchy and theta(13) with a remote detector of reactor antineutrinos," Phys. Rev. D 78 (2008) 071302 doi:10.1103/PhysRevD.78.071302 [hep-ex/0612022].

[7] L. Zhan, Y. Wang, J. Cao and L. Wen, "Determination of the Neutrino Mass Hierarchy at an Intermediate Baseline," Phys. Rev. D 78 (2008) 111103 doi:10.1103/PhysRevD.78.111103 [arXiv:0807.3203 [hep-ex]].

[8] L. Zhan, Y. Wang, J. Cao and L. Wen, "Experimental Requirements to Determine the Neutrino Mass Hierarchy Using Reactor Neutrinos,” Phys. Rev. D 79 (2009) 073007 doi:10.1103/PhysRevD.79.073007 [arXiv:0901.2976 [hep-ex]].

[9] O. Mena, I. Mocioiu and S. Razzaque, "Neutrino mass hierarchy extraction using atmospheric neutrinos in ice,” Phys. Rev. D 78 (2008) 093003 doi:10.1103/PhysRevD.78.093003 [arXiv:0803.3044 [hep-ph]].

[10] E. K. Akhmedov, S. Razzaque and A. Y. Smirnov, "Mass hierarchy, 2-3 mixing and CP-phase with Huge Atmospheric Neutrino Detectors,” JHEP 1302 (2013) 082 Erratum: [JHEP 1307 (2013) 026] doi:10.1007/JHEP02(2013)082, 10.1007/JHEP07(2013)026 [arXiv:1205.7071 [hep-ph]].

[11] F. An et al. [JUNO Collaboration], "Neutrino Physics with JUNO,” J. Phys. G 43 (2016) no.3, 030401 doi:10.1088/0954-3899/43/3/030401 [arXiv:1507.05613 [physics.ins-det]].

[12] S. Adrian-Martinez et al. [KM3Net Collaboration], "Letter of intent for KM3NeT 2.0," J. Phys. G 43 (2016) no.8, 084001 doi:10.1088/0954-3899/43/8/084001 [arXiv:1601.07459 [astro-ph.IM]].

[13] H. Nunokawa, S. J. Parke and J. W. F. Valle, “CP Violation and Neutrino Oscillations,” Prog. Part. Nucl. Phys. 60 (2008) 338 doi:10.1016/j.ppnp.2007.10.001 [arXiv:0710.0554 [hep-ph]].

[14] P. Coloma and E. Fernandez-Martinez, "Optimization of neutrino oscillation facilities for large $\theta_{13}$," JHEP 1204 (2012) 089 doi:10.1007/JHEP04(2012)089 [arXiv:1110.4583 [hep-ph]].

[15] S. Parke, "Neutrinos: Theory and Phenomenology," Phys. Scripta T 158 (2013) 014013 doi:10.1088/0031-8949/2013/T158/014013 [arXiv:1310.5992 [hep-ph]].

[16] T. R. Edgecock, O. Caretta, T. Davenne, C. Densham, M. Fitton, D. Kelliher, P. Loveridge and S. Machida et al., Phys. Rev. ST Accel. Beams 16 (2013) 021002 [arXiv:1305.4067 [physics.acc-ph]].

[17] The European Spallation Source, http://europeanspallationsource.se/, ESS TDR, Release 1.0, Nov. 2012.

[18] "A Very Intense Neutrino Super Beam Experiment for Leptonic CP Violation Discovery based on the European Spallation Source Linac”, Nuclear Physics B, Volume 885, August 2014, 127-149.

[19] A. de Bellefon, J. Bouchez, J. Busto, J. -E. Campagne, C. Cavata, J. Dolbeau, J. Dumarchez and P. Gorodetzky et al., hep-ex/0607026.

[20] L. Agostino et al. [MEMPHYS Collaboration], JCAP 1301 (2013) 024 [arXiv:1206.6665 [hep-ex]].

[21] Enrique Fernandez, private communication. 
[22] "nuSTORM - Neutrinos from STORed Muons: Letter of Intent to the Fermilab Physics Advisory Committee," arXiv:1206.0294 [hep-ex].

[23] COST Action CA15139 "Combining forces for a novel European facility for neutrino-antineutrino symmetry-violation discovery" (EuroNuNet)

http://euronunet.in2p3.fr/site/ 